\title{
Current-Time Characteristics of Resistive Superconducting Fault Current Limiters
}

\author{
Steven M. Blair, Student Member, IEEE, Campbell D. Booth, and Graeme M. Burt, Member, IEEE
}

\begin{abstract}
Superconducting fault current limiters (SFCLs) may play an important role in power-dense electrical systems. Therefore, it is important to understand the dynamic characteristics of SFCLs. This will allow the behavior of multiple SFCLs in a system to be fully understood during faults and other transient conditions, which will consequently permit the coordination of the SFCL devices to ensure that only the device(s) closest to the fault location will operate. It will also allow SFCL behavior and impact to be taken into account when coordinating network protection systems.

This paper demonstrates that resistive SFCLs have an inverse current-time characteristic: they will quench (become resistive) in a time that inversely depends upon the initial fault current magnitude. The timescales are shown to be much shorter than those typical for inverse overcurrent protection. A generic equation has been derived which allows the quench time to be estimated for a given prospective fault current magnitude and initial superconductor temperature, and for various superconducting device and material properties. This information will be of value to system designers in understanding the impact of SFCLs on network protection systems during faults, and planning the relative positions of multiple SFCLs.
\end{abstract}

Index Terms-Distributed generation, fault current limitation, low-carbon, power system protection, superconducting fault current limiter (SFCL).

\section{INTRODUCTION}

$\mathbf{S}$ UPERCONDUCTING fault current limiters (SFCLs) offer an attractive solution to the problem of rising fault levels in highly power-dense electrical systems [1], [2], [3], [4].

However, for SFCLs to be effectively applied in power systems, it is important to understand their transient properties in order to coordinate their operation with power system protection devices and to ensure that, in a multiple SFCL application, that only the SFCL(s) closest to the fault location operate in order to avoid unnecessary disturbance to healthy elements of the power system. This paper analyzes a typical transient SFCL model, and determines its current-time characteristics. Section II introduces the SFCL model, and the model is analyzed mathematically in Section III.

\section{SFCL MODEL}

\section{A. SFCL Model Requirements}

For this study, it is important to realistically model an SFCL's resistance characteristics, in particular:

This work was supported in part by the Engineering and Physical Sciences Research Council and in part by the Rolls-Royce Group plc.

Steven Blair, Campbell Booth, and Graeme Burt are with the Institute for Energy and Environment, Department of Electronic and Electrical Engineering, University of Strathclyde, Glasgow, G1 1XW, UK (e-mail: steven.blair@eee.strath.ac.uk).
- In a three-phase power system, each phase of the SFCL must be modeled independently because they will operate independently, particularly during unbalanced primary system faults, which represent the predominant mode of fault in power distribution systems (particularly in overhead systems) [5]. Each phase will have a dedicated superconducting wire (or several wires) which form a superconducting element. This means that within the first cycle of fault current during a three-phase to earth fault, each phase of the SFCL will develop resistance at a slightly different time, hence creating a momentary phase unbalance. Unbalanced faults may only cause a quench in only one or two phases of the SFCL. Independent operation of each phase must be represented such that the effects on the overall power system can be evaluated for all fault types at various locations.

- The SFCL model should be a reasonable approximation of transient SFCL behavior during faults, and therefore should consider thermal properties [6]. The model should be adaptable, with little or no modification, for different types of faults or other scenarios. It should be able to model the effects of different fault durations, different times of fault occurrences, different fault current magnitudes, and cater for any point of fault occurrence with respect to the voltage waveform. The model should execute without excessively long simulation times.

- The dimensions of the superconducting wires must be known. A given superconducting wire will have a full load current rating. To achieve a higher full load current rating, several wires may be connected in parallel, as described in [7], [8].

\section{B. SFCL Model Implementation}

SFCLs have been modeled using the equations from [9], which is based on a BSCCO (Bi2212) superconductor, and fits well with experimental results. Reference [10] extends this model with a simplified, yet practical, thermal sub-system. The model has been implemented in Simulink with SimPowerSystems [11]. An independent, single-phase superconductor model has been replicated to create a three-phase SFCL.

The model is based on the three possible states for a superconductor: flux-creep, flux-flow, and normal (resistive) conducting. Fig. 1 illustrates the typical relationship between electric field, $E$, and current density, $J$, for these three states. Table I defines the symbols used. Note that, after entering the flux-flow state, the superconductor heats up significantly, which in turn shifts the $E-J$ curve to the left; i.e., $E$ increases further. 
TABLE I

SUMMARY OF SFCL MODEL PARAMETERS

\begin{tabular}{|c|c|c|c|}
\hline Parameter description & Symbol & Value & Units \\
\hline Length of the superconductor wire & $l_{s c}$ & 50 & $\mathrm{~m}$ \\
\hline Diameter of the superconductor wire & $d_{s c}$ & $4.0 \times 10^{-3}$ & $\mathrm{~m}$ \\
\hline Cross-sectional area of the superconductor wire & $a_{s c}$ & $\pi\left(\frac{d_{s c}}{2}\right)^{2}$ & $\mathrm{~m}^{2}$ \\
\hline Superconductor temperature & $T(t)$ & - & $\mathrm{K}$ \\
\hline Superconductor current & $\overline{i_{s c}(t)}$ & - & A \\
\hline Ambient (starting) temperature of the superconductor and cooling reservoir & $T_{a}$ & 77 & $\mathrm{~K}$ \\
\hline Superconductor critical temperature & $T_{c}$ & 95 & $\mathrm{~K}$ \\
\hline Flux-creep region exponent (at $77 \mathrm{~K}$ ) & $n_{77 K}$ & 6 & - \\
\hline Flux-flow region exponent & $\beta$ & 3 & - \\
\hline Current density & $J(t)$ & $\frac{i_{s c}(t)}{a_{s c}}$ & $\mathrm{~A} / \mathrm{m}^{2}$ \\
\hline Critical current density (at $77 \mathrm{~K}$ ), i.e., current density where $E=1 \mu \mathrm{V} / \mathrm{cm}$ & $J_{c_{77 K}}$ & $1.5 \times 10^{7}$ & $\mathrm{~A} / \mathrm{m}^{2}$ \\
\hline Electric field at transition from flux-creep state to flux-flow state & $E_{0}$ & 0.1 & $\mathrm{~V} / \mathrm{m}$ \\
\hline Normal conducting state resistivity (at $T_{c}$ ) & $\rho$ & $1.0 \times 10^{-6}$ & $\Omega \mathrm{m}$ \\
\hline Coefficient for heat transfer to cooling reservoir & $\kappa$ & $1.5 \times 10^{3}$ & $\mathrm{~W} / \mathrm{Km}^{2}$ \\
\hline Superconductor volumetric specific heat & $c_{v}$ & $1.0 \times 10^{6}$ & $\mathrm{~J} / \mathrm{Km}^{3}$ \\
\hline Superconductor heat capacity & $c_{s c}$ & $l_{s c} a_{s c} c_{v}$ & $\mathrm{~J} / \mathrm{K}$ \\
\hline Thermal resistance from superconductor to cooling reservoir & $\theta_{s c}$ & $\frac{1}{\kappa l_{s c} \pi d_{s c}}$ & $\mathrm{~K} / \mathrm{W}$ \\
\hline Heat dissipated in the superconductor & $Q_{s c}(t)$ & $\int i_{s c}(t)^{2} R_{s c}(t) d t$ & $\mathrm{~J}$ \\
\hline Heat removed by the cooling system & $Q_{\text {removed }}(t)$ & $\int \frac{T(t)-T_{a}}{\theta_{s c}} d t$ & $\mathrm{~J}$ \\
\hline Instantaneous superconductor resistance & $R_{s c}(t)$ & $\frac{E(t, T) l_{s c}}{J(t) a_{s c}}$ & $\Omega$ \\
\hline
\end{tabular}

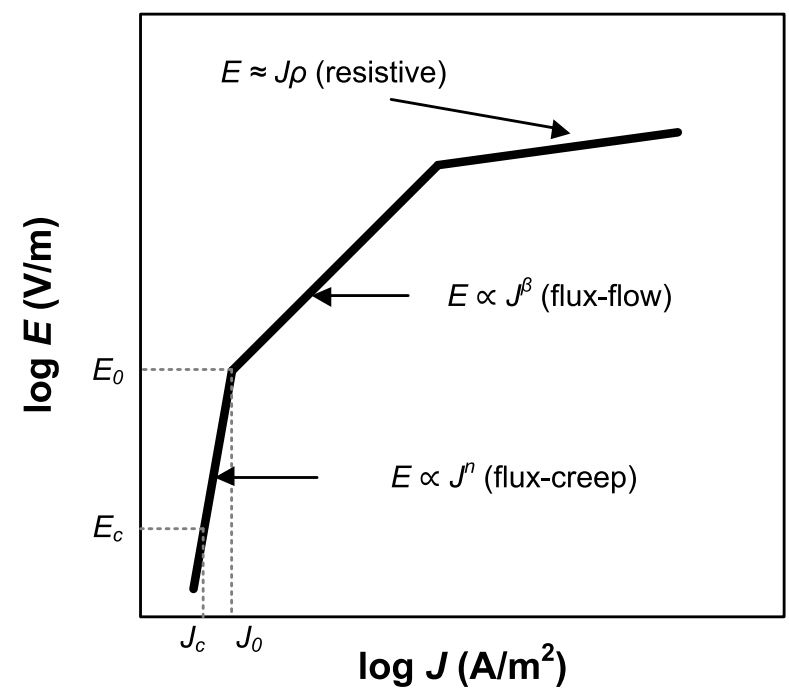

Fig. 1. Superconductor $E-J$ characteristic, showing three states (from [9])

The thermal sub-system has been modeled as follows:

$$
T(t)=T_{a}+\frac{1}{c_{s c}} \int_{0}^{t}\left[Q_{s c}(t)-Q_{\text {removed }}(t)\right] d t .
$$

It is assumed that the resistivity varies linearly with temperature, when $T(t) \geq T_{c}$, as given by (2). This is a reasonable assumption and is justified and supported by the experimental results in [12]. The variation of $J_{c}$ with temperature is also assumed to be linear, as shown in (3). Reference [13] illustrates this property for $\mathrm{BSCCO}$, and reference [14] for $\mathrm{MgB}_{2}$; it is this variation of $J_{c}$ (and $J_{0}$ ) with temperature which allows the operating current for an SFCL to be adjusted.

$$
\begin{gathered}
E(t, T) \approx \rho\left(\frac{T}{T_{c}}\right) J(t), \quad T(t) \geq T_{c} \\
J_{c}(T) \approx J_{c_{77 K}}\left(\frac{T_{c}-T(t)}{T_{c}-77}\right)
\end{gathered}
$$

Many of the parameter values given in Table I are taken from [10]. The resistivity at $T_{c}, \rho$, was selected as $1 \mu \Omega \mathrm{m}$ and the length, $l_{s c}$, was varied to limit fault current to approximately the same root-mean-square (RMS) value as load current. This results in an SFCL phase resistance of approximately $4.0 \Omega$ at $95 \mathrm{~K}$, and $12.6 \Omega$ at $300 \mathrm{~K}$. The diameter, $d_{s c}$, was chosen such that the superconductor does not enter the flux-flow state during normal load current, but that each SFCL will enter the flux-flow state during the first cycle of fault current. In reality, the wire diameter may be fixed, but several wires may be connected in parallel to achieve a particular current rating [8]. $\beta$ was reduced to 3 to reduce the rate of increase of flux-flow resistance. A cylindrical superconductor wire geometry is used, rather than the cuboid shape modeled in [10]. 


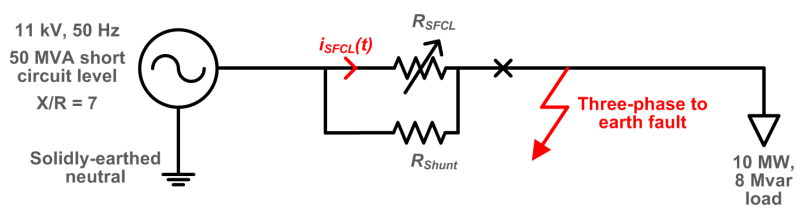

Fig. 2. SFCL fault current limitation test network
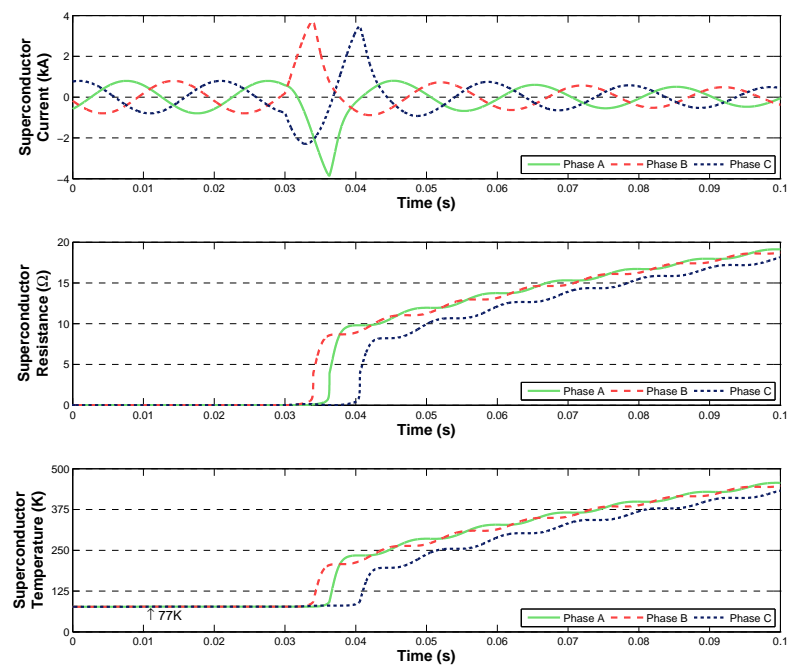

Fig. 3. SFCL model response for a three-phase to earth fault

\section{SFCL Model Example}

To test the fault current limitation properties of the model, the circuit in Fig. 2 was simulated. Fig. 3 illustrates the response of the model for a three-phase to earth fault, with negligible fault resistance. The SFCL quenches in each phase during the first peak of fault current. If the fault is cleared 0.1 $\mathrm{s}$ after occurrence, each phase of the SFCL takes between 2 $\mathrm{s}$ and $4 \mathrm{~s}$ to drop below $T_{c}$. This SFCL design corresponds to the "slow-heating" category described in [9], because the quench time is in the order of $10 \mathrm{~ms}$.

Resistive SFCLs typically incorporate an impedance that is connected electrically in parallel with the SFCL [1], [8], [10], as shown in Fig. 2. To simplify the analysis in this paper, it is assumed that there is no shunt impedance. The effect of the shunt impedance will be most prominent after an SFCL fully quenches to the resistive state, which does not affect the analysis in the following section.

\section{ANALysis of SFCL CURRENT-Time CHARACTERISTICS}

\section{A. Analytical Solution}

The SFCL model equations can be analyzed to approximate the current-time grading, i.e., the time taken to quench for a given fault current. The temperature of the superconductor is calculated using (1), where $E(t, T)$ (within $Q_{s c}(t)$ ) is calculated according to [9], in the flux-flow region (where $E(t, T) \geq E_{0}$ and $\left.T(t)<T_{c}\right)$. When $T(t) \geq T_{c}$, it is assumed that the superconductor quenches. Equation (1) can be differentiated and manipulated as follows:

$$
\begin{aligned}
\frac{d T}{d t} & =\frac{1}{c_{s c}}\left[Q_{s c}(t)-Q_{\text {removed }}(t)\right] \\
\frac{d T}{d t} & =\frac{1}{c_{s c}}\left[i(t) E(t, T) l_{s c}-\frac{T(t)-T_{a}}{\theta_{s c}}\right] \\
d t & =\frac{d T}{\frac{1}{c_{s c}}\left[i(t) E(t, T) l_{s c}-\frac{T(t)-T_{a}}{\theta_{s c}}\right]} .
\end{aligned}
$$

To simplify the analysis, a constant dc fault current, $I$, is assumed. Therefore, $E$ can be simplified to be only a function of temperature:

$$
\int d t=\int \frac{d T}{\frac{1}{c_{s c}}\left[I E(T) l_{s c}-\frac{T(t)-T_{a}}{\theta_{s c}}\right]}
$$

where $E(T)$ is [9]:

$$
E(T)=E_{0}\left(\frac{E_{c}}{E_{0}}\right)^{\frac{\beta}{n_{77 K}}}\left(\frac{J_{c_{77 K}}}{J_{c}(T)}\right)\left(\frac{J(t)}{J_{c_{77 K}}}\right)^{\beta} .
$$

Substituting (3) into (5) gives:

$$
\begin{aligned}
E(T) & =E_{0}\left(\frac{E_{c}}{E_{0}}\right)^{\frac{\beta}{n_{77 K}}}\left(\frac{T_{c}-77}{T_{c}-T}\right)\left(\frac{J(t)}{J_{c_{77 K}}}\right)^{\beta} \\
& =E_{0}\left(\frac{E_{c}}{E_{0}}\right)^{\frac{\beta}{n_{77 K}}}\left(\frac{T_{c}-77}{T_{c}-T}\right)\left(\frac{I}{J_{c_{77 K}} a_{s c}}\right)^{\beta} .
\end{aligned}
$$

For convenience, the non-temperature-dependent part of $E(T)$, along with $I$ and $l_{s c}$ from (4), can be combined as $k$ as follows:

$$
\begin{aligned}
I E(T) l_{s c} & =k \frac{T_{c}-77}{T_{c}-T} \\
k & =I E_{0}\left(\frac{E_{c}}{E_{0}}\right)^{\frac{\beta}{n_{77 K}}}\left(\frac{I}{J_{c_{77 K} a_{s c}}}\right)^{\beta} l_{s c} \\
k & =E_{0}\left(\frac{E_{c}}{E_{0}}\right)^{\frac{\beta}{n_{77 K}}} \frac{I^{\beta+1}}{\left(J_{c 77 K} a_{s c}\right)^{\beta}} l_{s c .}
\end{aligned}
$$

This provides an expression for $t$, the time for the superconductor to reach a particular temperature, $T$, as follows:

$$
t=c_{s c} \int \frac{d T}{\left[k \frac{T_{c}-77}{T_{c}-T}-\frac{T-T_{a}}{\theta_{s c}}\right]} .
$$

Equation (6) can be solved by (8). The constant, $C$, given in (9), can be calculated by substituting $T(0)=T_{a}$ into (8).

In the common case, using liquid nitrogen as the cryogen, where $T_{a}=77 \mathrm{~K}, T_{c}=95 \mathrm{~K}$, and $T=95 \mathrm{~K}$, (8) can be simplified to (7) as follows:

$$
t=6 c_{s c} \theta_{s c} \frac{\arctan \left(\frac{3}{\sqrt{2 \theta_{s c}-9}}\right)}{\sqrt{2 \theta_{s c} k-9}} .
$$

Fig. 4 illustrates the current-time characteristics for three different values of the initial temperature, $T_{a}$. For example, for $T_{a}=77 \mathrm{~K}$, a fault current of at least $1.9 \mathrm{kA}$ (RMS or 


$$
\begin{gathered}
t=c_{s c} \theta_{s c}\left(\frac{\arctan \left(\frac{T_{a}-2 T+T_{c}}{\sqrt{-\left(T_{a}-T_{c}\right)^{2}+4 \theta_{s c}\left(T_{c}-77\right)}}\right)\left(T_{a}-T_{c}\right)}{\sqrt{-\left(T_{a}-T_{c}\right)^{2}+4 \theta_{s c} k\left(T_{c}-77\right)}}-\frac{\ln \left(T^{2}+\left(-T_{a}-T_{c}\right) T+T_{a} T_{c}+\theta_{s c} k\left(T_{c}-77\right)\right)}{2}\right)+C \\
C=c_{s c} \theta_{s c}\left(\frac{\arctan \left(\frac{T_{a}-T_{c}}{\sqrt{-\left(T_{a}-T_{c}\right)^{2}+4 \theta_{s c} k\left(T_{c}-77\right)}}\right)\left(T_{a}-T_{c}\right)}{\sqrt{-\left(T_{a}-T_{c}\right)^{2}+4 \theta_{s c} k\left(T_{c}-77\right)}}+\frac{\ln \left(\theta_{s c} k\left(T_{c}-77\right)\right)}{2}\right)
\end{gathered}
$$

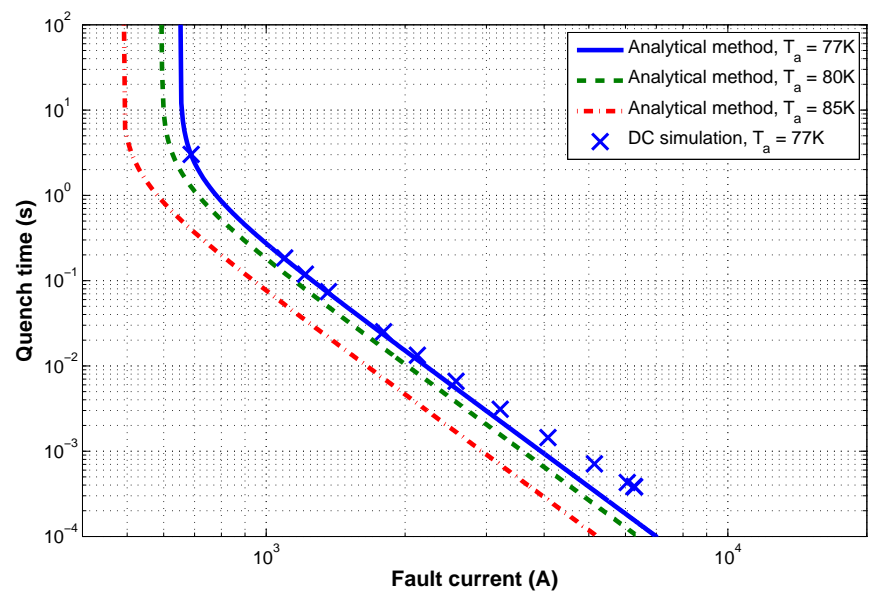

Fig. 4. SFCL model current-time characteristics (analysis and simulation). Fault current for dc simulation is the initial fault current from a constant dc voltage source.

dc) is required to ensure SFCL quenching within the first ac cycle $(20 \mathrm{~ms})$, compared with $1.4 \mathrm{kA}$ when $T_{a}=85 \mathrm{~K}$. The cut-off current for which quenching would not occur is shown by the vertical asymptotes. It can be noted that varying the initial superconductor temperature, $T_{a}$, affects both the critical current and the position with respect to the y-axis of the current-time curve, as illustrated in Fig. 4. Although this analysis excludes factors such as the instantaneous values of ac current and the point on wave of fault inception (see Section III-B), it does show that an SFCL will inherently act in a manner that is consistent with an inverse current-time graded protection system.

It should be noted that, unlike inverse current-time protection relays, the flux-flow resistance will reduce fault current before a circuit breaker opens, thereby slightly delaying quenching, compared with the quench time predicted by (7). For simplicity, this is ignored in the analysis above, but Fig. 4 also illustrates simulation results, using a single-phase constant dc voltage source. The fault current only drops by approximately $1-5 \%$, until very close to the quench point. Furthermore, very short quench times, in the order of $1 \mathrm{~ms}$ or less, may be problematic in practice because of the potential for transient overvoltages due to the high rate of change of current through the circuit inductance, $L$, with voltage given by $L \frac{d i}{d t}[9]$.

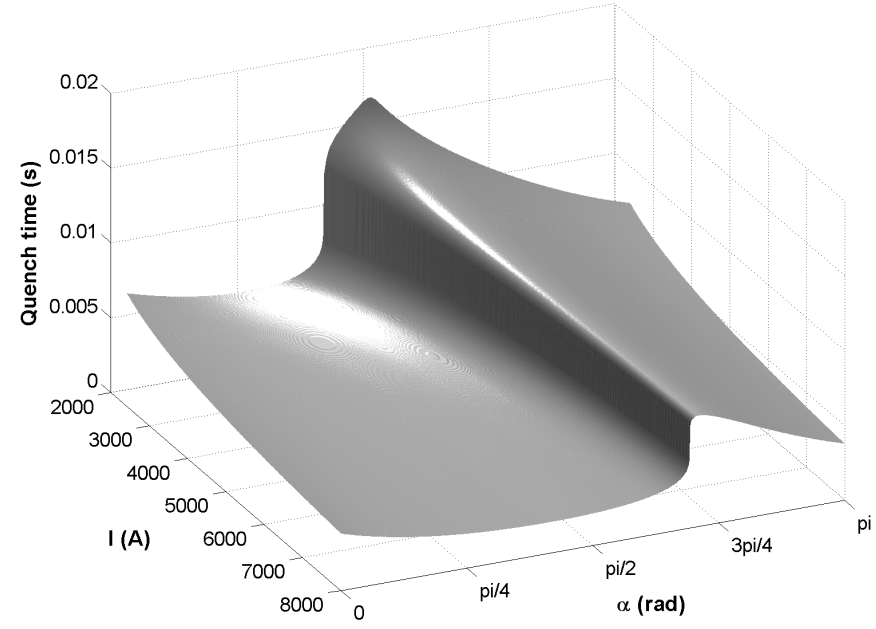

Fig. 5. Effect of current magnitude and $\alpha$ on quench time

\section{B. Effect of the ac Point on Wave of Fault Inception}

The instantaneous ac current during a fault can be modeled using (10) as follows [8]:

$$
i(t)=I\left(\sin (\omega t+\alpha-\phi)-\sin (\alpha-\phi) e^{\frac{-R t}{L}}\right)
$$

where $I$ is the current magnitude, $\omega=2 \pi f, f=50 \mathrm{~Hz}, \alpha$ is the point on wave of fault inception, and $\phi=\arctan \left(\frac{\omega L}{R}\right)=$ $\arctan \left(\frac{X}{R}\right)$. The $\mathrm{X} / \mathrm{R}$ ratio is kept constant with a value of 7. Fig. 5 illustrates how $I$ and $\alpha$ affect the quench time. The point of wave can delay a quench by $5-10 \mathrm{~ms}$, depending on the current magnitude. The sharp transition between $\pi / 2$ and $3 \pi / 4$ is due to the combination of dc offset and current phase $(\alpha-\phi)$ which results in a relatively small area under the current curve (and therefore low energy dissipation in the superconductor) during the first half cycle. If quenching does not occur within the first half-cycle of fault current, then there is a relatively long delay until quenching may occur at some point during the second half-cycle. SFCLs must therefore be carefully applied such that quenching will occur within the first cycle of fault current (at the required fault current magnitude), for any possible point on wave of fault inception.

\section{Comparison of SFCL and Overcurrent Protection Current- Time Characteristics}

To place the SFCL current-time curves in Fig. 4 into perspective, they can be compared with an equivalent inverse 


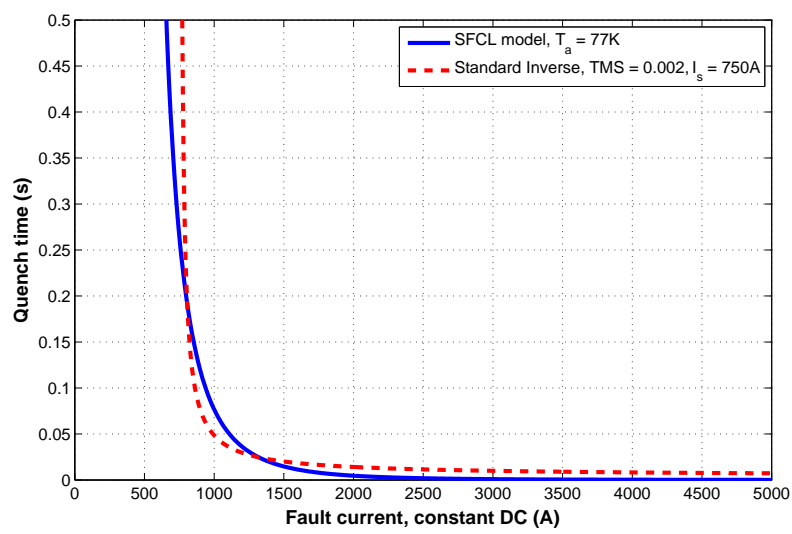

Fig. 6. SFCL model current-time characteristics vs. Standard Inverse curve

definite minimum time (IDMT) curve, commonly used for overcurrent protection. Fig. 6 compares the SFCL characteristics with the Standard Inverse curve [5], given by (11):

$$
t=T M S \frac{0.14}{I_{r}{ }^{0.02}-1}
$$

where TMS is the time multiplier setting, $I_{r}=\frac{I}{I_{s}}$, and $I_{s}$ is the setting current. For Fig. $6, T M S=0.002$ and $I_{s}=750$ A. Clearly, the curves differ, especially at higher currents, but this does demonstrate that an SFCL has similar characteristics to IDMT protection, albeit with a $T M S$ value that would be extremely small in practice. This information could be used to coordinate the operation of multiple SFCLs in a power system in a similar fashion to the method used to coordinate overcurrent protection relays.

\section{CONCLUSIONS}

This paper has established, through analysis of an existing SFCL model, that resistive SFCLs have an inverse currenttime characteristic. This has been verified mathematically. The analysis approach represents a generic design tool which could help with the following:

- The coordination of multiple SFCLs, especially if they have different designs, e.g., from different manufacturers.

- Quickly investigating the effect that modifying parameters, such as superconductor length, diameter, etc., has on the current-time characteristics and the critical current value.

- As a guide for adjusting the superconductor temperature, while the SFCL is in service, to cater for different system conditions and fault current reduction requirements.

- To determine the required superconductor volume for a desired current-time characteristic (i.e., solving for $k$, or a particular component of $k$ ).

Note that these results are closely linked to this particular SFCL model; nevertheless, the technique is valid as long as the SFCL characteristics are well known. Further work is needed to examine the coordination of multiple SFCLs, and the implications for existing protection systems.

\section{REFERENCES}

[1] M. Noe and M. Steurer, "High-temperature superconductor fault current limiters: concepts, applications, and development status," Superconductor Science and Technology, vol. 20, no. 3, pp. R15-R29, Mar. 2007.

[2] S. Blair, N. Singh, C. Booth, and G. Burt, "Operational control and protection implications of fault current limitation in distribution networks," in Universities Power Engineering Conference (UPEC), 2009 Proceedings of the 44th International, 2009, pp. 1-5.

[3] N. K. Singh, R. M. Tumilty, G. M. Burt, C. G. Bright, C. C. Brozio, D. A. Roberts, A. C. Smith, and M. Husband, "System-Level Studies of a $\mathrm{MgB} 2$ Superconducting Fault-Current Limiter in an Active Distribution Network," IEEE Trans. Appl. Supercond., vol. 20, no. 2, pp. 54-60, Apr. 2010.

[4] S. Blair, C. Booth, I. Elders, N. Singh, G. Burt, and J. McCarthy, "Superconducting fault current limiter application in a power-dense marine electrical system," IET Electrical Systems in Transportation, vol. 1, no. 3, p. 93, 2011.

[5] Alstom Grid, Network Protection \& Automation Guide. Alstom Grid, 2011.

[6] I. N. Dul'kin, D. V. Yevsin, L. M. Fisher, V. P. Ivanov, A. V. Kalinov, and V. A. Sidorov, "Modeling Thermal Process in a Resistive Element of a Fault Current Limiter," IEEE Trans. Appl. Supercond., vol. 18, no. 1, pp. 7-13, Mar. 2008.

[7] R. Dommerque, S. Krämer, A. Hobl, R. Böhm, M. Bludau, J. Bock, D. Klaus, H. Piereder, A. Wilson, T. Krüger, G. Pfeiffer, K. Pfeiffer, and S. Elschner, "First commercial medium voltage superconducting fault-current limiters: production, test and installation," Superconductor Science and Technology, vol. 23, no. 3, p. 034020, Mar. 2010.

[8] S. M. Blair, C. D. Booth, N. K. Singh, G. M. Burt, and C. G. Bright, "Analysis of Energy Dissipation in Resistive Superconducting FaultCurrent Limiters for Optimal Power System Performance," IEEE Trans. Appl. Supercond., vol. 21, no. 4, pp. 3452-3457, Aug. 2011.

[9] W. Paul, M. Chen, M. Lakner, J. Rhyner, D. Braun, W. Lanz, and M. Kleimaier, "Superconducting fault current limiter: applications, technical and economical benefits, simulations and test results," CIGRE SC 13, Tech. Rep., 2000.

[10] J. Langston, M. Steurer, S. Woodruff, T. Baldwin, and J. Tang, “A generic real-time computer Simulation model for Superconducting fault current limiters and its application in system protection studies," IEEE Trans. Appl. Supercond., vol. 15, no. 2, pp. 2090-2093, 2005.

[11] MathWorks, "Real-Time Workshop - Generate C code from Simulink models and MATLAB code - Simulink," 2011. [Online]. Available: http://www.mathworks.com/products/rtw/

[12] S. L. Liu, G. Longyan, B. Gang, W. Haiyun, and L. Yongtao, "The temperature dependence of the resistivity in Ba $1-$ x K x Fe 2 As 2 superconductors," Superconductor Science and Technology, vol. 24, no. 7, p. 075005 , Jul. 2011

[13] S. Kozak, T. Janowski, B. Kondratowicz-Kucewicz, J. Kozak, and G. Wojtasiewicz, "Experimental and Numerical Analysis of Energy Losses in Resistive SFCL," IEEE Trans. Appl. Supercond., vol. 15, no. 2, pp. 2098-2101, Jun. 2005.

[14] A. Oliver, A. Smith, M. Husband, M. Bailey, and Y. Feng, "Assessment of Small Bend Diameter Magnesium Diboride Wire for a Superconducting Fault Current Limiter Application," IEEE Trans. Appl. Supercond., vol. 19 , no. 3, pp. 1942-1945, 2009.

Steven M. Blair (S'09) received the M.Eng. degree (with distinction) in Computer and Electronic Systems in 2008 from the University of Strathclyde, Glasgow, U.K., where he is currently pursuing the Ph.D. degree in electrical engineering.

His research interests include power system protection, fault current limitation, marine electrical systems, communications, and real-time simulation.

Campbell D. Booth received the B.Eng. and Ph.D. degrees in electrical and electronic engineering from the University of Strathclyde, Glasgow, U.K, in 1991 and 1996, respectively. He is currently a Senior Lecturer with the Institute for Energy and Environment, Department of Electronic and Electrical Engineering, University of Strathclyde. 
His research interests include power system protection; plant condition monitoring and intelligent asset management; applications of intelligent system techniques to power system monitoring, protection, and control; knowledge management; and decision support systems.

Graeme M. Burt (M'95) received the B.Eng. degree in electrical and electronic engineering and the Ph.D. degree following research into fault diagnostic techniques for power networks from the University of Strathclyde, Glasgow, U.K., in 1988 and 1992, respectively.

$\mathrm{He}$ is currently the Director of the Institute for Energy and Environment, University of Strathclyde, where he also directs the University Technology Center in Electrical Power Systems sponsored by the Rolls-Royce Group plc. $\mathrm{He}$ is a professor of electrical power engineering, and has particular research interests in the areas of: integration of distributed generation; power system modelling and real-time simulation; power system protection and control; microgrids and more-electric systems. 